\title{
Transcriptome and miRNA network analysis of familial hypercholesterolemia
}

\author{
${\text { HAIBIN } \mathrm{CHEN}^{1 *}, \text { LIANG WANG }}^{2}$ and JINFA JIANG ${ }^{1 *}$ \\ ${ }^{1}$ Department of Medical Cardiology, Tongji Hospital, Tongji University, \\ Shanghai 200065; ${ }^{2}$ Department of Cardiovascular Surgery, The General Hospital of \\ Ningxia Medical University, Yinchuan, Ningxia 750004, P.R. China
}

Received August 4, 2013; Accepted November 14, 2013

DOI: $10.3892 /$ ijmm.2013.1610

\begin{abstract}
Familial hypercholesterolemia (FH) is a genetic disorder characterized by a high serum concentration of low-density lipoprotein (LDL) cholesterol. The high LDL cholesterol level leads to an excess deposition of cholesterol in the arterial walls and accelerated atherosclerosis, thereby increasing the risk of premature coronary heart disease. In the present study, we used a DNA microarray approach to identify gene expression profiles that distinguish patients with $\mathrm{FH}$ from healthy control subjects. Furthermore, transcription factors (TFs), microRNAs (miRNAs), target genes and pathways were analyzed to explore the potential transcriptional interactions occurring in FH. Publicly available microarray and regulation data were used to construct a regulatory network to identify additional genes related to $\mathrm{FH}$ and their interactions. The results revealed that specificity protein 1 (SP1), signal transducer and activator of transcription 1 (STAT1) and spleen focus forming virus (SFFV) proviral integration oncogene spil (SPI1) play a central role in the $\mathrm{FH}$ regulatory network. In addition, the TF, upstream transcription factor 2, c-fos interacting (USF2) and the gene, Wiskott-Aldrich syndrome (WAS), were identified to be associated with $\mathrm{FH}$, although no reports for these proteins exist in the literature. Overall, transcriptional network analysis proved to be effective approach to identify novel targets for FH therapy.
\end{abstract}

\section{Introduction}

Familial hypercholesterolemia (FH) is a genetic disorder characterized by a high serum concentration of low-density lipoprotein (LDL) cholesterol. The high LDL cholesterol level accelerates the deposition of cholesterol in the arterial walls

Correspondence to: Dr Jinfa Jiang, Department of Medical Cardiology, Tongji Hospital, Tongji University, 389 Xincun Road, Putuo, Shanghai 200065, P.R. China

E-mail: jiangjinfajinfa@hotmail.com

*Contributed equally

Key words: familial hypercholesterolemia, target genes, microarray analysis and leads to atherosclerosis, thereby increasing the risk of premature coronary heart disease (1).

The most common genetic defects underlying $\mathrm{FH}$ are mutations in the LDL receptor (LDLR), the apolipoprotein $B$ (ApoB)-100 and the pro-protein convertase subtilisin/kexin type 9 (PCSK9) genes (2). LDL cholesterol normally circulates in the body for 2.5 days, and subsequently binds to LDLR on liver cells, undergoes endocytosis and is then digested. LDL is removed, and the synthesis of cholesterol by the liver is suppressed by the HMG-CoA reductase pathway. In FH, the function of LDLR is reduced or absent, and LDL circulates for an average duration of 4.5 days, resulting in a significantly increased level of LDL cholesterol in the blood, while other lipoproteins show normal levels (3). In patients with ApoB mutations, the reduced binding of LDL particles to the receptor causes an increased level of LDL cholesterol (4). The expression of PCSK9 normally downregulates the LDLR pathway by indirectly inducing the degradation of the LDLR protein, and loss-of-function mutations in PCSK9 result in low plasma LDL levels. Thus, PCSK9 is an attractive target for the development of novel drugs aiming to reduce the serum LDL cholesterol level (5).

DNA microarray analysis is a high-throughput approach that allows the comparison of transcriptional profiles between healthy and diseased individuals or samples, among other applications (6). DNA microarray analysis has been previously used to analyze gene expression patterns and to identify differentially expressed genes in $\mathrm{FH}$ (7).

In this study, we used a similar strategy to identify gene expression profiles that distinguish patients with $\mathrm{FH}$ from healthy control subjects. Furthermore, data on the relevant transcription factors (TFs), microRNAs (miRNAs), target genes and pathways were analyzed so as to construct an $\mathrm{FH}$ regulatory network and to explore the potential molecular interactions involved in the development of $\mathrm{FH}$.

\section{Materials and methods}

Data resources

Affymetrix microarray data. The transcription profiles of patients with ischemic cardiomyopathy (8) were obtained from the study with Accession no. GSE6054 deposited at the GEO 
Table I. Regulation data form TRANSFAC and TRED.

\begin{tabular}{lcccc}
\hline Source & No. of interactions & TFs & No. of target genes & Link \\
\hline TRANSFAC & 774 & 219 & 265 & http:/www.gene-regulation.com/pub/databases.html \\
TRED & 5,722 & 102 & 2,920 & http://rulai.cshl.edu/TRED \\
Total & 6,328 & 276 & 3,002 & \\
\hline
\end{tabular}

TRANSFAC, Transcription Factor Database; TRED, Transcriptional Regulatory Element Database; TFs, transcription factors.

database (http://www.ncbi.nlm.nih.gov/geo). RNA samples from 6 patients with heterozygous $\mathrm{FH}$ and 13 healthy controls were hybridized onto the Affymetrix Human Genome U133 Plus 2.0 Array.

Pathway data. The Kyoto Encyclopedia of Genes and Genomes (KEGG) is an online database collection gathering information on genomes, enzymatic pathways and biological compounds or drugs, among others (9). The KEGG pathway database lists molecular interaction networks and their organism-specific variants (http://www.genome.jp/kegg). A total of 130 pathways, involving 2,287 genes, was collected from the KEGG pathway.

Regulation data. There are approximately 2,600 proteins in the human genome that contain DNA-binding domains, and most of these are presumed to function as TFs (10). The combined use of a subset of approximately 2,000 human TFs easily accounts for the unique regulation of each gene in the human genome during development (11). These TFs are grouped into 5 superfamilies, based on the presence of conserved DNA-binding domains. The TRANScription FACtor database (TRANSFAC) contains data on TFs, their experimentally proven binding sites, and regulated genes (12). The Transcriptional Regulatory Element Database (TRED) has been built in response to increasing needs for an integrated repository for both cis- and trans-regulatory elements in mammals (13). TRED contains curated information on transcriptional regulation, including experimentally verified $\mathrm{TF}$ binding motifs. We collected 774 pairs of regulatory interactions between 219 TFs and 265 target genes from TRANSFAC and 5,722 pairs of regulatory interactions between 102 TFs and 2,920 target genes from TRED. By combining the two datasets, a total of 6,328 regulatory interactions between 276 TFs and 3,002 target genes were collected (Table I).

miRNA datasets. We integrated disease-associated miRNA datasets with datasets of miRNAs and target genes to explore the association between miRNA-regulated genes and diseases. The human miRNA disease database (HMDD) (14), a resource of curated information on the associations of miRNAs and disease as supported by experimental evidence, contains 444 miRNA genes, 259 diseases, 1,149 publications and 2,886 miRNA-disease associations. miR2Disease (15) provides a comprehensive resource of miRNA deregulation in various human diseases. A total of 349 miRNAs and 163 diseases were collected from this database. The above 2 databases were merged, resulting in 5,036 associations of miRNAs with diseases. We also integrated experimentally supported data on miRNA target genes from starBase (16), miRecords (17) and
TarBase (18). Overall, 211,464 associations between miRNA and target genes were selected.

\section{Methods}

Differentially expressed gene (DEG) analysis. For the GSE6054 dataset, the limma method (19) was used to identify DEGs. The raw expression data from all conditions were normalized using the RMA method implemented in Bioconductor (20) with default settings, and then a linear model was applied. DEGs were defined as genes showing a fold change value $>1.5$ and a corrected p-value $<0.05$.

Co-expression analysis. To demonstrate the potential regulatory association between TFs and DEGs, Pearson's correlation coefficient (PCC) was calculated for all pair-wise comparisons of gene expression values between the TFs and DEG. The regulatory association was considered significant at $\mathrm{PCC}>0.6$ or $\mathrm{PCC}<-0.6$.

Gene Ontology (GO) analysis. DAVID (21), a highthroughput and integrated data-mining environment, analyzes gene lists derived from transcriptomic comparisons. We used DAVID to identify categories of biological processes (as defined in the GO annotation system) that were overrepresented in our dataset, by applying a threshold p-value $<0.01$.

Construction of regulatlory network between genes and TFs. Using the regulation data from TRANSFAC and TRED, we matched differentially expressed TFs to their predicted target genes. Based on this, and information on the interactions between genes in the pathways they belong to, we constructed regulatory networks using Cytoscape (22). Regulatory associations between TFs and genes were verified based on co-expression analysis.

Pathway significance analysis. We adopted an impact approach that calculates the statistical significance of a set of genes belonging to a certain pathway, but also considers other factors, such as the magnitude of change in expression of each gene, the topology of the related pathway and interactions between the genes (23). In this model, the impact factor $(I F)$ of a pathway $P_{i}$ is calculated as the sum of 2 terms (equation 1) as follows:

$$
I F(P i)=\log \left(\frac{1}{p i}\right)+\frac{\sum_{g \in P i}|P F(g)|}{|\Delta E| \cdot N_{d k}(P i)}
$$

The first term is a probabilistic term that captures the significance of the given pathway $P_{i}$ from the perspective of the set of genes in the pathway. It is obtained by using a hyper- 
Table II. Significance pathway analysis, .

\begin{tabular}{lccc}
\hline $\begin{array}{l}\text { Pathway } \\
\text { name }\end{array}$ & $\begin{array}{c}\text { Impact } \\
\text { factor }(I F)\end{array}$ & $\begin{array}{c}\text { \% DEGs } \\
\text { in pathway }\end{array}$ & $\begin{array}{c}\text { Corrected } \gamma \\
\text { p-value }\end{array}$ \\
\hline Long-term depression & 19.586 & 2.667 & $6.42 \mathrm{E}-08$ \\
Gap junction & 13.185 & 6.250 & $2.66 \mathrm{E}-05$ \\
Long-term potentiation & 12.560 & 4.110 & $4.76 \mathrm{E}-05$ \\
Apoptosis & 11.736 & 10.112 & $1.02 \mathrm{E}-04$ \\
B cell receptor signaling pathway & 11.166 & 6.154 & $1.72 \mathrm{E}-04$ \\
Focal adhesion & 10.414 & 3.448 & $3.43 \mathrm{E}-04$ \\
Melanoma & 8.827 & 5.634 & 0.001442 \\
Adherence junction & 7.888 & 5.128 & 0.003335 \\
Regulation of actin, cytoskeleton & 7.814 & 2.304 & 0.003561 \\
MAPK signaling pathway & 7.488 & 4.412 & 0.004751 \\
\hline
\end{tabular}

Pathway terms are derived from KEGG. DEGs, differentially expressed genes.

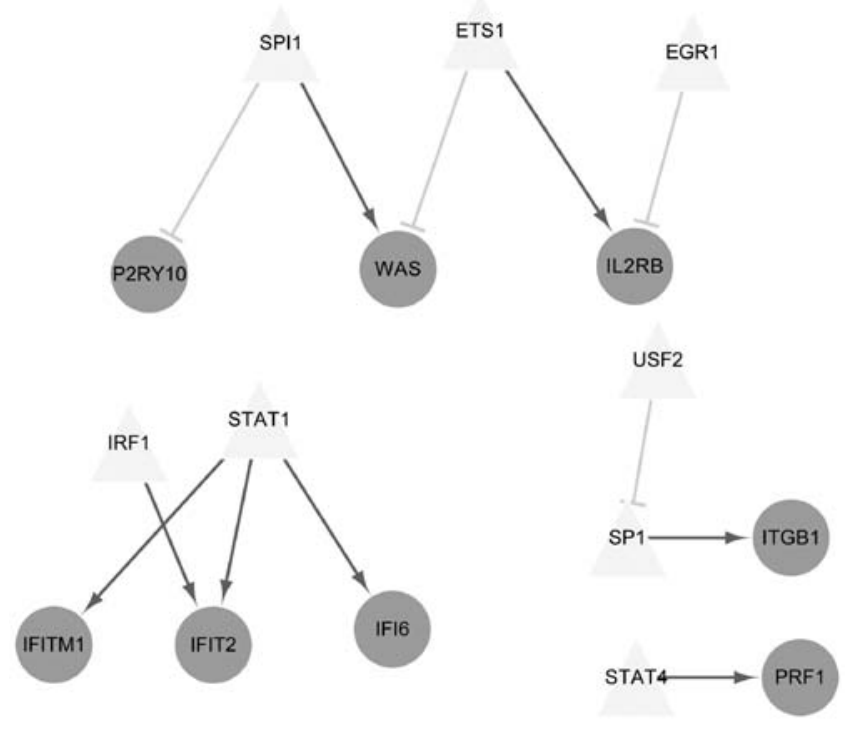

Figure 1. Regulatory network in familial hypercholesterolemia (FH).

geometric model in which $p_{i}$ is the probability of obtaining at least the observed number of DEGs, $N_{d e}$ by chance $(24,25)$. The second term is a functional term, that depends on the identity of the specific genes that are differentially expressed, as well as on the interactions described by the pathway (i.e., its topology). The second term sums up the absolute values of the perturbation factor $(P F)$ for all genes ' $g$ ' on the given pathway $P_{i}$. The $P F$ of a gene ' $g$ ' is calculated (equation 2 ) as follows:

$$
P F(g)=\Delta E(g)+\sum_{u \in U S g} \beta_{u g} \cdot \frac{P F(u)}{N_{d s}(u)}
$$

In this equation, the term $\Delta E(g)$ captures the quantitative information measured in the gene expression experiment. The factor $\Delta E(g)$ represents the normalized measured expression change of the gene ' $g$ '. The second term is the sum of all $P F s$ of the genes ' $u$ ' directly upstream of the target gene ' $g$ ' $P F(u)$, normalized by the number of downstream genes for each such gene $N_{d s}(u)$, and weighted by a factor $\beta_{u g}$, which reflects the type of interaction: $\beta_{u g}=1$ for induction and $\beta_{u g}=-1$ for repression (KEGG supplies this information in the description of the pathway topology). $U S_{g}$ is the set of all genes upstream of ' $g$ '. In equation 1 , we normalized for the size of the pathway by dividing the total perturbation by the number of DEGs in the given pathway, $N_{d e}\left(P_{i}\right)$. In order to make the $I F$ calculation as independent as possible from the technology and also comparable between studies (23), we also divided the second term in equation 1 by the mean absolute fold change $\triangle E$, calculated across all DEGs. The results from the pathway significance analysis are presented in Table II.

Construction of a regulatory network between TFs and pathways. To further investigate the regulatory association between TFs and different pathways, we mapped DEGs to pathways and constructed a regulatory network describing the associations between the TFs predicted to regulate these genes and pathways.

miRNA network construction. From the miRNA-disease data collected from the HMDD and miR2Disease databases, we focused on those concerning esophageal diseases. The esophageal disease-related miRNAs were then mapped to their predicted target genes. In total, 14 miRNAs and 431 DEGs were used to construct the network.

\section{Results}

Regulatory network in FH. To obtain a list of genes differentially expressed in $\mathrm{FH}$, we downloaded raw data from the publicly available microarray dataset, GSE6054, from the GEO repository. Following data normalization, 443 DEGs were identified based on a fold change value threshold $>1.5$ and a $\mathrm{p}$-value threshold $<0.05$. To obtain the regulatory associations between these DEGs and TFs, we applied a PCC threshold $>0.6$ in the co-expression analysis. In total, 12 regulatory associations were identified, involving $8 \mathrm{TFs}$ and 8 differentially expressed target genes. The resulting regulatory network describing FH is presented in Fig. 1. In this network, specificity protein 1 (SP1), signal transducer and 
Table III. Top 10 biological processes (BP) enriched according to DAVID analysis of Gene Ontology (GO) terms.

\begin{tabular}{|c|c|c|c|}
\hline GO term category & Count & p-value & FDR \\
\hline 0010628-positive regulation of gene expression & 7 & $1.37 \mathrm{E}-05$ & 0.018446 \\
\hline 0045893-positive regulation of transcription, DNA-dependent & 6 & $8.21 \mathrm{E}-05$ & 0.110665 \\
\hline 0051254-positive regulation of RNA metabolic process & 6 & $8.54 \mathrm{E}-05$ & 0.11514 \\
\hline 0010604-positive regulation of macromolecule metabolic process & 7 & $1.23 \mathrm{E}-04$ & 0.165523 \\
\hline 0045941-positive regulation of transcription & 6 & $1.81 \mathrm{E}-04$ & 0.244052 \\
\hline $\begin{array}{l}\text { 0045935-positive regulation of nucleobase, nucleoside, nucleotide } \\
\text { and nucleic acid metabolic process }\end{array}$ & 6 & $2.91 \mathrm{E}-04$ & 0.391377 \\
\hline 0051173-positive regulation of nitrogen compound metabolic process & 6 & $3.37 \mathrm{E}-04$ & 0.453138 \\
\hline 0010557-positive regulation of macromolecule biosynthetic process & 6 & $3.61 \mathrm{E}-04$ & 0.486679 \\
\hline $\begin{array}{l}\text { 0045944-positive regulation of transcription from } \\
\text { RNA polymerase II promoter }\end{array}$ & 5 & $4.48 \mathrm{E}-04$ & 0.602755 \\
\hline 0031328-positive regulation of cellular biosynthetic process & 6 & 4.48E-04 & 0.602793 \\
\hline
\end{tabular}

FDR, false discovery rate.

activator of transcription 1 (STAT1) and spleen focus forming virus (SFFV) proviral integration oncogene spi1 (SPI1) formed local networks with a higher node degree, which suggests that these genes play important roles in FH. STAT1 activates 3 target genes, interferon induced transmembrane protein 1 (IFITM1), interferon-induced protein with tetratricopeptide repeats 2 (IFIT2) and interferon, $\alpha$-inducible protein 6 (IFI6). $\mathrm{SP} 1$, regulated by upstream transcription factor 2, c-fos interacting (USF2), activates integrin, beta 1 (ITGB1), while the Wiskott-Aldrich syndrome (WAS) gene is regulated by both transcription factors, SPI1 and v-ets erythroblastosis virus E26 oncogene homolog 1 (avian) (ETS1).

GO analysis of the regulatory network in FH. Several GO categories were enriched among the 16 genes in the regulatory network. These included biological processes (BP) such as positive regulation of gene expression, positive regulation of transcription, DNA-dependent positive regulation of RNA metabolic process etc. (Table III lists the top 10 enriched BP categories).

Pathways involved in FH. To identify the pathways that are the most affected by $\mathrm{FH}$, we used a statistical approach at the pathway level. Significance analysis at the single gene level may suffer from the limited number of samples and from experimental noise, which can severely limit the power of the selected statistical test. Pathway analysis allows the application of a more relaxed significance threshold than the one applied to single genes, while still leading to better biological interpretations. Therefore, we adopted a pathwaybased impact analysis method that considered a number of factors, including the statistical significance of changes in the expression of DEGs that are assigned to a certain pathway, the magnitude of change in expression of each gene, the topology of the pathway, and the predicted regulatory interactions among genes. The impact analysis revealed a number of path- ways deemed relevant to $\mathrm{FH}$, such as long-term depression, gap junction and long-term potentiation (Table II).

Regulatory network of TFs and pathways. To further investigate the regulatory associations between TFs and pathways, we assigned DEGs to the relevant pathways and obtained a regulatory network between TFs regulating these DEGs (based on the co-expression analysis results) and pathways (Fig. 2). In this network, SP1, USF2 and STAT4 were identified as hub nodes linked to a high number of FH-related pathways. For example, STAT4 activates type I diabetes mellitus, STAT1 activates the B cell receptor signaling pathway, while among pathways regulated by SP1 were those related to focal adhesion and axon guidance.

miRNA network in FH. Using the miRNA-disease data collected from the HMDD and miR2Disease databases, we matched the genes previously identified as part of the $\mathrm{FH}$ regulation nework to data describing the miRNA-target gene associations. The FH-related miRNAs were selected and mapped to disease-miRNA data. A total of 132 miRNAs and 16 genes were used to construct the network. The final miRNA network includes five genes associated with 37 miRNAs with node degrees $>2$ (Fig. 3).

\section{Discussion}

The present analysis allowed the identification of a number of TFs and pathways associated with FH. Among these, SP1, STAT1 and SPI1 formed a local network, which suggests that these genes play important roles in FH. In addition, SP1, USF2 and STAT4 appeared as hub nodes in the TFs-pathways network. We discuss below the association between FH and the genes identified herein based on the current literature.

SP1, a human transcription factor, contains a zinc finger protein motif, by which it binds directly to DNA and enhances 


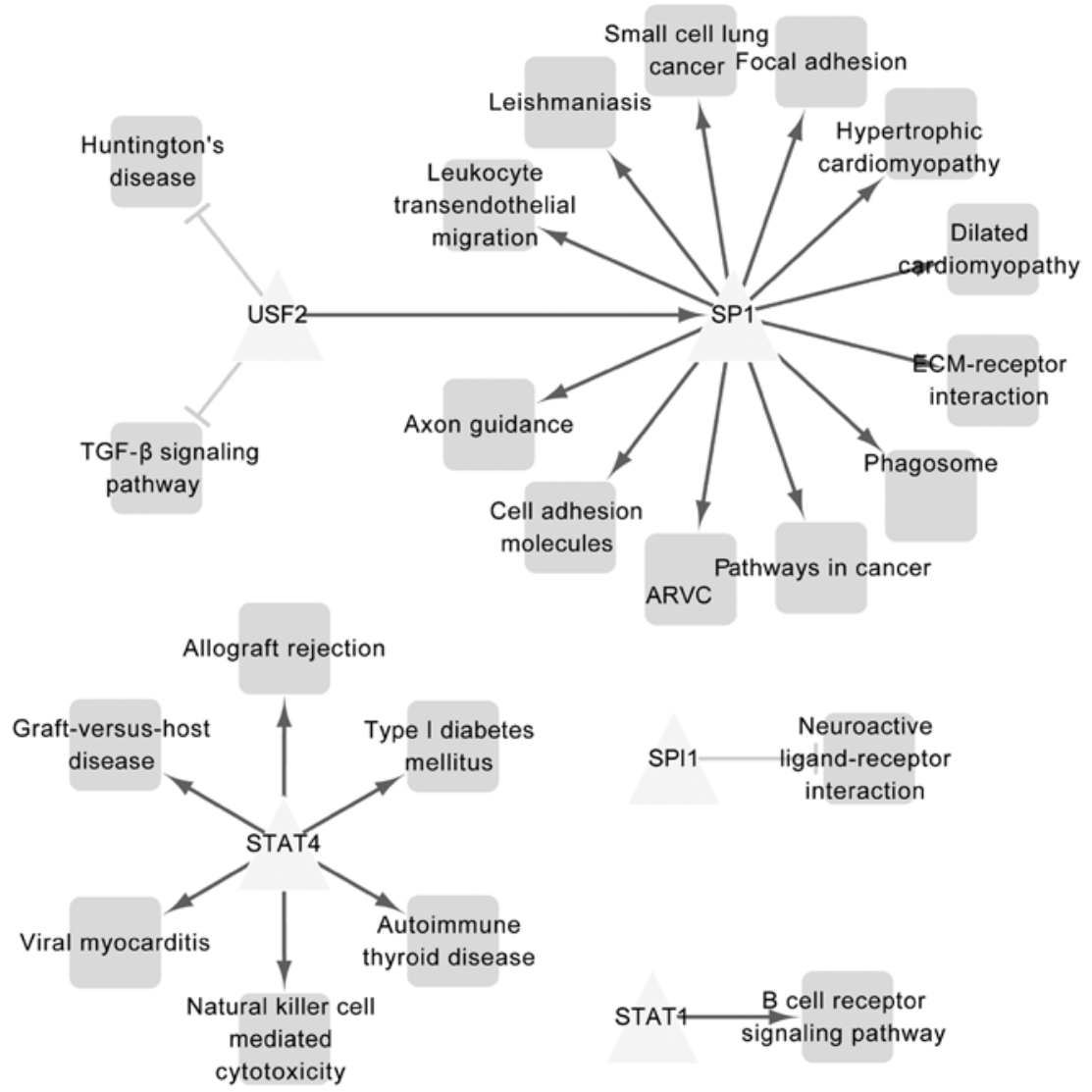

Figure 2. Regulatory network of transcription factors (TFs) and pathways in familial hypercholesterolemia (FH).

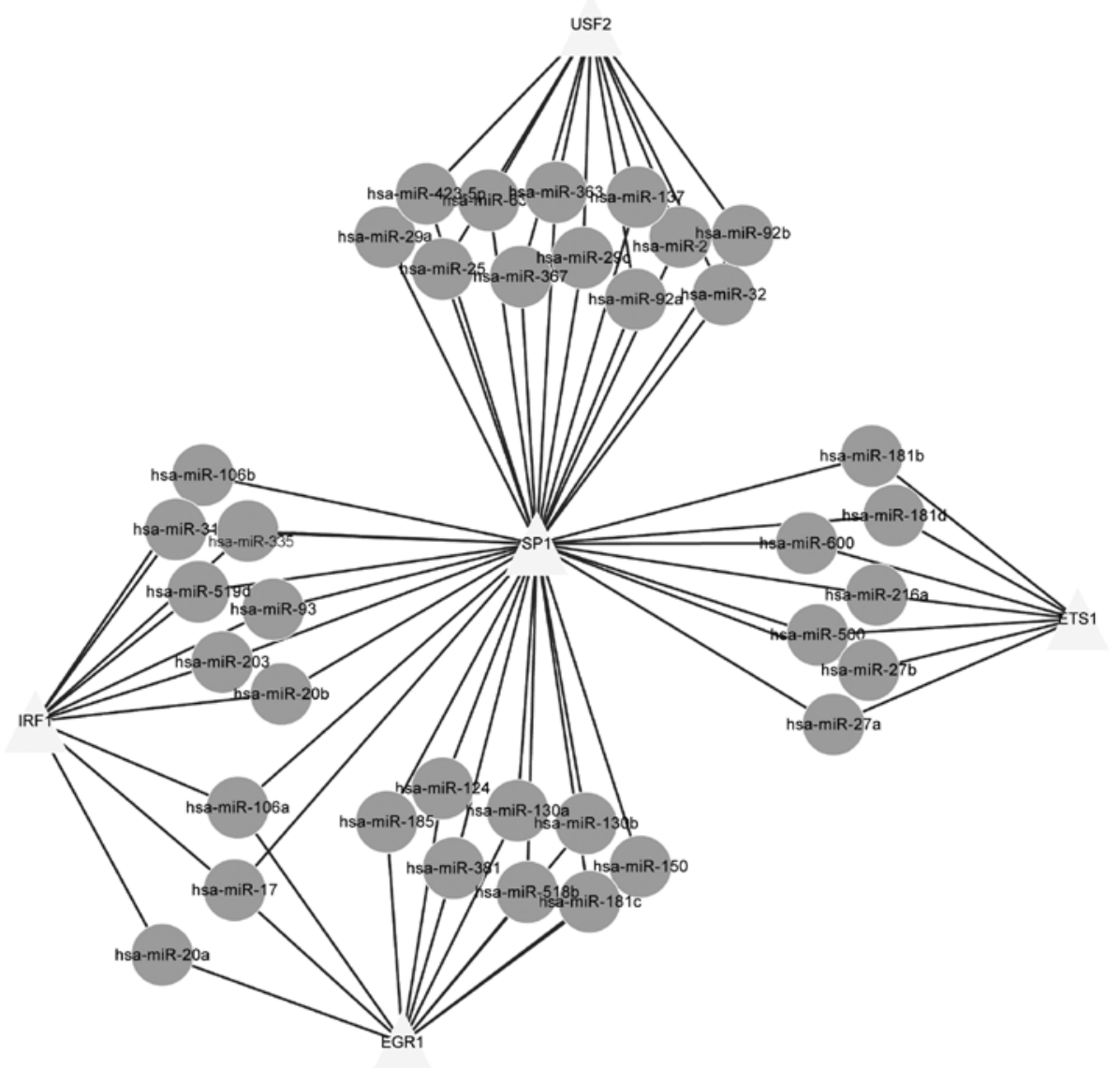

Figure 3. miRNA network in familial hypercholesterolemia (FH). 
gene transcription. A rare mutation (T-45C) was identified in the LDLR gene in a patient with FH. The mutation lies on the proximal SP1 binding site, in repeat 3 of the 42-bp region of the promoter required for sterol-dependent regulation of transcription. The substituted nucleotide is not a strongly conserved base of the SP1 binding site, and this mutation reduced SP1 binding affinity and led to $\mathrm{FH}$ (26). In addition, two more mutations in the LDLR gene, $-49 \mathrm{C}>\mathrm{T}$ and $-139 \mathrm{C}>\mathrm{G}$, have been identified, with one of these shown to induce the loss of SP1 binding in a patient with FH $(27,28)$.

The SPI1 gene encodes an ETS-domain transcription factor that activates gene expression during myeloid and B-lymphoid cell development. It is also known as PU.1. A previous study showed that $12 / 15$-lipoxygenase $(12 / 15$-LO) plays a role in promoting atherogenesis in a mouse model of $\mathrm{FH} .12 / 15-\mathrm{LO}^{-/-}$ macrophages exhibited defective nuclear expression of interferon consensus sequence-binding protein (ICSBP). In conjunction with c-Rel and PU.1, ICSBP has been shown to form a multiprotein complex, which binds to the ETS-2 site of the interleukin (IL)-12 p40 promoter. The inhibition of this complex led to the selective decrease of IL-12 p40 in macrophages and to the reduction of atherosclerotic lesions (29).

STAT1 is a member of the STAT protein family. This protein can be activated by various ligands, including interferon (IFN)- $\alpha$, IFN- $\gamma$, epidermal growth factor (EGF), platelet-derived growth factor (PDGF) and IL-6, to function as a transcriptional activator. Analysis of monocytes from patients with $\mathrm{FH}$ revealed that a significant number of these have elevated STAT1 levels, as a result of activation by IFN- $\gamma$ (30).

The ITGB1 gene encodes a $\beta$ subunit of integrins. Integrin family members are membrane receptors involved in cell adhesion and recognition in a variety of processes. Monocyte activation and migration into the arterial wall, with subsequent differentiation into macrophages, are key events in atherogenesis associated with FH. ITGB1 has been found to be highly expressed during monocyte to macrophage differentiation and differentially expressed in $\mathrm{CD}_{16}{ }^{-}$and $\mathrm{CD} 16^{+}$monocytes, indicating a later stage of maturation of $\mathrm{CD}^{+} 6^{+}$monocytes from FH homozygotes compared with monocytes from healthy individuals (31). This protein may play an important role in monocyte adhesion and the development of atherosclerosis, thereby representing a potentially novel therapeutic target for atherosclerosis (32).

Autopsy studies in children have shown that atherosclerotic lesions begin to develop first in the intima of the aorta. Therefore, a previous study examined the feasibility of measuring intimamedial thickness of the abdominal aorta (aIMT) in children and studied its value in distinguishing high-risk children from healthy controls as compared to a more established marker of subclinical atherosclerosis, the common carotid artery intimamedial thickness (cIMT). The results indicated that children with FH and type 1 diabetes show increased IMT compared with healthy controls, with a relatively greater increase in aIMT than in cIMT. These data suggested that aIMT may provide the best currently available noninvasive marker of preclinical atherosclerosis and type 1 diabetes in children (33).

A number of studies have indicated that $\mathrm{T}$ and $\mathrm{B}$ cells exert a pro-atherogenic effect in FH. Cell-mediated immune responses by macrophages and $\mathrm{T}$ lymphocytes occur in atherosclerotic lesions. These are to a large extent caused by specific
$\mathrm{CD}^{+} \mathrm{T}$ cells responding to oxidized LDK (ox-LDL) (34). Ox-LDL is an important local antigen in atherosclerosis and recognized by antigen receptors, such as $\mathrm{T}$ cell receptors (TCRs). Such local T cell responses may activate B cells through B cell receptors (BCRs), with concomitant systemic antibody production (35).

In conclusion, we used network analysis as an exploratory framework to study the pathobiology of $\mathrm{FH}$, based on the assumption that $\mathrm{FH}$ is a contextual attribute of distinct interaction patterns among multiple genes. The most notable results of our study include the identification of FH-related TFs, target genes and pathways such as SP1, SPI1, STAT1, ITGB1, focal adhesion pathway, type I diabetes mellitus pathway and $\mathrm{B}$ cell receptor signaling pathway. In addition, our results indicated that USF2 and WAS might play an important role in $\mathrm{FH}$, although no relevant report for this finding exists in the literature. Therefore, future studies are required to confirm our results.

\section{References}

1. Jansen A, van Aalst-Cohen ES, Tanck MWT, et al: Genetic determinants of cardiovascular disease risk in familial hypercholesterolemia. Arterioscl Thromb Vasc Biol 25: 1475-1481, 2005.

2. Rader DJ, Cohen J and Hobbs HH: Monogenic hypercholesterolemia: new insights in pathogenesis and treatment. J Clin Invest 111: 1795-1803, 2003.

3. Durrington P: Dyslipidaemia. Lancet 362: 717-731, 2003.

4. Castillo S, Tejedor D, Mozas P, et al: The apolipoprotein B R3500Q gene mutation in Spanish subjects with a clinical diagnosis of familial hypercholesterolemia. Atherosclerosis 165 : $127-135,2002$.

5. Soutar AK and Naoumova RP: Mechanisms of disease: genetic causes of familial hypercholesterolemia. Nat Clin Pract Cardiovasc Med 4: 214-225, 2007.

6. Spies M, Dasu MR, Svrakic N, et al: Gene expression analysis in burn wounds of rats. Am J Physiol Regul Integr Comp Physiol 283: R918-R930, 2002.

7. Dušková L, Kopečková L, Jansová E, et al: An APEX-based genotyping microarray for the screening of 168 mutations associated with familial hypercholesterolemia. Atherosclerosis 216: 139-145, 2011.

8. Mosig S, Rennert K, Buttner P, et al: Monocytes of patients with familial hypercholesterolemia show alterations in cholesterol metabolism. BMC Med Genomics 1: 60, 2008.

9. Kanehisa M and Goto S: KEGG: Kyoto Encyclopedia of Genes and genomes. Nucleic Acids Res 28: 27-30, 2000.

10. Wachi S, Yoneda K and Wu R: Interactome-transcriptome analysis reveals the high centrality of genes differentially expressed in lung cancer tissues. Bioinformatics 21: 4205-4208, 2005.

11. Brivanlou AH and Darnell JE Jr: Signal transduction and the control of gene expression. Science 295: 813-818, 2002.

12. Wingender E: The TRANSFAC project as an example of framework technology that supports the analysis of genomic regulation. Brief Bioinform 9: 326-332, 2008.

13. Jiang $C, X$ uan $Z$, Zhao $F$ and Zhang MQ: TRED: a transcriptional regulatory element database, new entries and other development. Nucleic Acids Res 35: D137-D140, 2007.

14. Lu M, Zhang Q, Deng M, et al: An analysis of human microRNA and disease associations. PLoS One 3: e3420, 2008.

15. Jiang Q, Wang Y, Hao Y, et al: miR2Disease: a manually curated database for microRNA deregulation in human disease. Nucleic Acids Res 37: D98-D104, 2009.

16. Yang JH, Li JH, Shao P, Zhou H, Chen YQ and Qu LH: starBase: a database for exploring microRNA-mRNA interaction maps from Argonaute CLIP-Seq and Degradome-Seq data. Nucleic Acids Res 39: D202-D209, 2011.

17. Xiao F, Zuo Z, Cai G, Kang S, Gao X and Li T: miRecords: an integrated resource for microRNA-target interactions. Nucleic Acids Res 37: D105-D110, 2009.

18. Papadopoulos GL, Reczko M, Simossis VA, Sethupathy P and Hatzigeorgiou AG: The database of experimentally supported targets: a functional update of TarBase. Nucleic Acids Res 37: D155-D158, 2009. 
19. Smyth GK: Linear models and empirical bayes methods for assessing differential expression in microarray experiments. Stat Appl Genet Mol Biol 3: Article3, 2004.

20. Gentleman RC, Carey VJ, Bates DM, et al: Bioconductor: open software development for computational biology and bioinformatics. Genome Biol 5: R80, 2004.

21. Huang da W, Sherman BT and Lempicki RA: Systematic and integrative analysis of large gene lists using DAVID bioinformatics resources. Nat Protoc 4: 44-57, 2009.

22. Shannon P, Markiel A, Ozier O, et al: Cytoscape: a software environment for integrated models of biomolecular interaction networks. Genome Res 13: 2498-2504, 2003.

23. Draghici S, Khatri P, Tarca AL, et al: A systems biology approach for pathway level analysis. Genome Res 17: 1537-1545, 2007.

24. Tavazoie S, Hughes JD, Campbell MJ, Cho RJ and Church GM Systematic determination of genetic network architecture. Nat Genet 22: 281-285, 1999.

25. Draghici S, Khatri P, Martins RP, Ostermeier GC and Krawetz SA: Global functional profiling of gene expression. Genomics 81: 98-104, 2003.

26. Sun XM, Neuwirth C, Wade DP, Knight BL and Soutar AK: A mutation (T-45C) in the promoter region of the low-density-lipoprotein (LDL)-receptor gene is associated with a mild clinical phenotype in a patient with heterozygous familial hypercholesterolaemia (FH). Hum Mol Genet 4: 2125-2129, 1995.

27. Mozas P, Galetto R, Albajar M, Ros E, Pocovi M and Rodriguez-Rey JC: A mutation $(-49 \mathrm{C}>\mathrm{T})$ in the promoter of the low density lipoprotein receptor gene associated with familial hypercholesterolemia. J Lipid Res 43: 13-18, 2002.
28. Smith AJ, Ahmed F, Nair D, et al: A functional mutation in the LDLR promoter $(-139 \mathrm{C}>\mathrm{G})$ in a patient with familial hypercholesterolemia. Eur J Hum Genet 15: 1186-1189, 2007.

29. Zhao L, Cuff CA, Moss E, et al: Selective interleukin-12 synthesis defect in 12/15-lipoxygenase-deficient macrophages associated with reduced atherosclerosis in a mouse model of familial hypercholesterolemia. J Biol Chem 277: 35350-35356, 2002.

30. Grewal T, Boudreau M, Roy M, et al: Expression of gamma-IFN responsive genes in scavenger receptor over-expressing monocytes is associated with xanthomatosis. Atherosclerosis 138: 335-345, 1998.

31. Mosig S, Rennert K, Krause S, et al: Different functions of monocyte subsets in familial hypercholesterolemia: potential function of $\mathrm{CD} 14^{+} \mathrm{CD} 16^{+}$monocytes in detoxification of oxidized LDL. FASEB J 23: 866-874, 2009.

32. Wu H, Gower RM, Wang H, et al: Functional role of CD11 $\mathrm{c}^{+}$ monocytes in atherogenesis associated with hypercholesterolemia. Circulation 119: 2708-2717, 2009.

33. Jarvisalo MJ, Jartti L, Nanto-Salonen K, et al: Increased aortic intima-media thickness: a marker of preclinical atherosclerosis in high-risk children. Circulation 104: 2943-2947, 2001.

34. Hulthe J, Wikstrand J, Lidell A, Wendelhag I, Hansson GK and Wiklund O: Antibody titers against oxidized LDL are not elevated in patients with familial hypercholesterolemia. Arterioscler Thromb Vasc Biol 18: 1203-1211, 1998.

35. Binder CJ, Chang MK, Shaw PX, et al: Innate and acquired immunity in atherogenesis. Nat Med 8: 1218-1226, 2002. 